\title{
Prevalence of chronic kidney disease and cardiovascular comorbidities in adults in First Nations communities in northwest Ontario: a retrospective observational study
}

\author{
Len Kelly MD MClinSci, Cai-lei Matsumoto MPH, Yoko Schreiber MD MSc, Janet Gordon PND, \\ Hannah Willms, Christopher Olivier MD, Sharen Madden MD MSc, Josh Hopko, \\ Sheldon W. Tobe MD MScCH
}

\section{Abstract}

Background: The prevalence of adult chronic kidney disease and cardiovascular comorbidities in Canadian Indigenous communities is largely unknown. We conducted a study to document the prevalence of chronic kidney disease and concurrent diabetes mellitus, hypertension and dyslipidemia in a First Nations population in northwest Ontario.

Methods: In this observational study, we used retrospective data collected from regional electronic medical records of 16170 adults (age $\geq 18 \mathrm{yr}$ ) from 26 First Nations communities in northwest Ontario from May 2014 to May 2017. Demographic and laboratory data included age, gender, prescribed medications, estimated glomerular filtration rate, urine albumin:creatinine ratio, low-density lipoprotein cholesterol (LDL-C) level and glycated hemoglobin $\left(\mathrm{HbA}_{1 c}\right)$ concentration. We identified patients with diabetes by an $\mathrm{HbA}_{1 c}$ concentration of $6.5 \%$ or higher, or the use of a diabetic medication, those with dyslipidemia by an elevated LDL-C level ( $\geq 2.0 \mathrm{mmol} / \mathrm{L}$ ) or use of lipid-lowering medication, and those with hypertension by use of antihypertensive medication.

Results: Of the 16170 adults residing in the communities, 5224 unique patients (32.3\%) had renal testing (albumin:creatinine ratio and/or estimated glomerular filtration rate). The age-adjusted prevalence of chronic kidney disease was $14.5 \%$, and the prevalence of stage 3-5 chronic kidney disease (estimated glomerular filtration rate $<60 \mathrm{~mL} / \mathrm{min}$ ) was $7.0 \%$. Most patients with chronic kidney disease (1487 [80.0\%]) had at least 1 cardiovascular comorbidity. A total of 1332 patients $(71.6 \%)$ had diabetes, $1313(70.6 \%)$ had dyslipidemia, and 1098 (59.1\%) had hypertension; all 3 comorbidities were present in 716 patients (38.5\%).

Interpretation: We document a high prevalence of advanced chronic kidney disease in this First Nations population, $7.0 \%$, double the rate in the general population. High rates of cardiovascular comorbidities were also common in these patients with chronic kidney disease, which places them at increased risk for cardiovascular disease.

$\mathrm{P}$ lanning chronic disease management services for a population requires knowledge of the existing comorbid diseases to assist in both primary and secondary prevention. For rural and remote populations spread over a vast region who must come to a central health hub for services such as dialysis initiation, it is essential. Information on the prevalence of chronic kidney disease, a precursor to endstage renal disease, and its risk factors for progression is a necessity given the impact on quality of life and costs involved. ${ }^{1-3}$ In response to rising rates of end-stage renal disease and chronic kidney disease, First Nations community chiefs in northwest Ontario requested a better understanding of the burden of chronic kidney disease and related comorbidities.

Indigenous Canadians experience a high burden of chronic kidney disease. ${ }^{4-6}$ A 2001 screening study in Canadian First Nations communities suggested an estimate in adults of up to $30 \% .{ }^{6}$ First Nations populations in Canada are more likely than
non-Indigenous populations to progress to end-stage renal disease, and their rate of death related to chronic kidney disease is $77 \%$ higher than that in the non-Indigenous population..$^{7-10}$ The prevalence of diabetes mellitus, a major risk for progressive chronic kidney disease, is higher in Indigenous population than in non-Indigenous populations, and it occurs at a younger age and is associated with increased mortality, cardiovascular disease and lower limb amputation. ${ }^{11,12}$ Uncontrolled hypertension is also major risk factor for progression to end-stage renal disease and, together with diabetes, dyslipidemia and chronic

\section{Competing interests: None declared.}

This article has been peer reviewed.

Correspondence to: Len Kelly, Ikelly@mcmaster.ca

CMAJ Open 2019. DOI:10.9778/cmajo.20190040 
kidney disease, contributes to a high rate and severity of cardiovascular disease, the leading cause of death in this population. ${ }^{13,14}$ The purpose of this study was to document the prevalence of chronic kidney disease and concurrent diabetes, hypertension and dyslipidemia in a First Nations population in northwest Ontario.

\section{Methods}

\section{Setting}

The setting was northwest Ontario, where 26 remote First Nations communities are supported in primary care by the Sioux Lookout First Nations Health Authority, which oversaw the study (Figure 1).

\section{Design}

For this descriptive study, we used retrospective clinical data from a regional electronic medical record system from May 2014 to May 2017, during which most patients with a chronic condition had a primary care encounter. Most patients with chronic conditions have at least 1 primary care visit in 2 years. ${ }^{15,16}$ We chose a 3 -year period to capture a robust number of patients with relevant prescriptions or investigations to estimate both incident and prevalent cases. Thirty community physicians have used the OSCAR (Open Source Clinical Application and Resource) electronic medical record system exclusively since 2013. The OSCAR database includes all community members accessing primary care in their community or in Sioux Lookout. All primary care visits, medications and investigations are recorded in the electronic medical record. ${ }^{17}$ The study population comprised all patients aged 18 years or more who had at least 1 clinical encounter between May 2014 and May 2017 and received a prescription or investigation for renal function, diabetes, hypertension or dyslipidemia.

\section{Sources of data}

Data were extracted by the Sioux Lookout First Nations Health Authority information technology department (J.H.) and verified by its public health service epidemiologist (C.M.). Data services are overseen by an information management

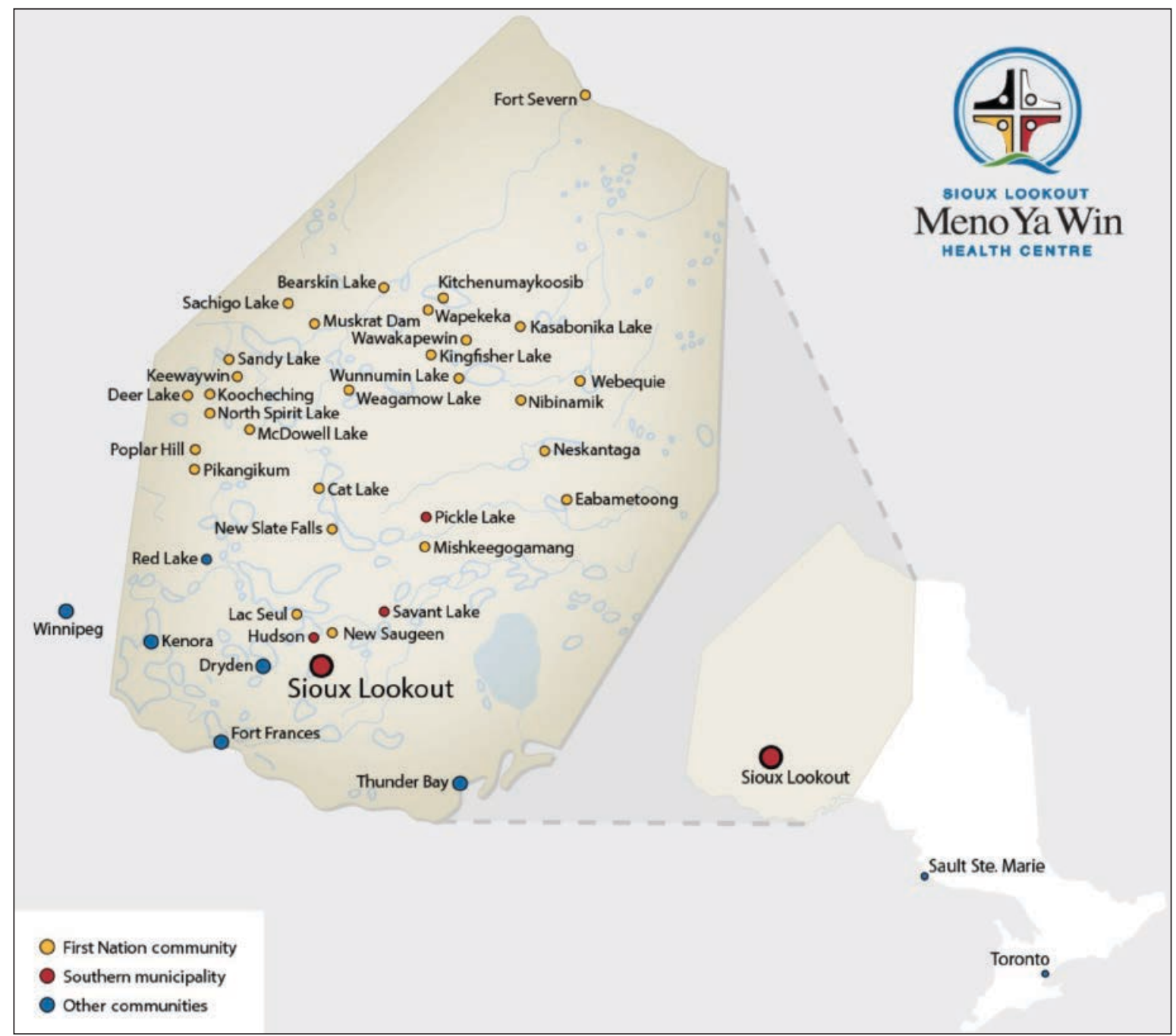

Figure 1: Sioux Lookout First Nations Health Authority and neighbouring communities in northwestern Ontario. Reproduced with permission of the Sioux Lookout Meno Ya Win Health Centre. 
committee of the Sioux Lookout Regional Physicians Services. Data collection followed Canadian Primary Care Sentinel Surveillance Network methods and validated case definition; the network also provided online support for local data extraction. ${ }^{18,19}$

Clinical data in the OSCAR electronic medical record are input by individual health care providers at the time of patient visits. Laboratory data are automatically updated by the Sioux Lookout Meno Ya Win Health Centre laboratory, which preforms all inpatient and outpatient testing. Medication data in the electronic medical record are regularly updated by the 30 treating physicians and are linked to regional pharmacies. Outpatient prescribing is done exclusively through the electronic medical record by regional primary care physicians. The extracted data accurately reproduced patient chart information in 20 randomly selected charts, several from either data subset (diabetes medications, low-density lipoprotein cholesterol [LDL-C] values). We used Anatomic Therapeutic Classification $\operatorname{codes}^{20}$ to select all disease-related medication data (antihypertensive medications [C02], peripheral vasodilators [C04], $\beta$-blocking agents [C07], calcium-channel blockers [C08], agents acting on the renin-angiotensin system [C09], lipid-modifying agents [C10A-B], insulin and analogues [A10A] and blood-glucose-lowering drugs [A10B]).

Diagnostic codes were not available as they are not automatically recorded in the electronic medical record. Laboratory investigations and medication prescribing were believed to be complete; they can be performed only through the electronic medical record system and were thought to be a reliable reflection of disease prevalence. ${ }^{18}$

Demographic and laboratory data included age, gender, medications, estimated glomerular filtration rate, albumin: creatinine ratio, LDL-C level and glycated hemoglobin $\left(\mathrm{HbA}_{1 \mathrm{c}}\right)$. The most recent laboratory value was used. We defined chronic kidney disease according to International Society of Nephrology criteria: estimated glomerular filtration rate less than $60 \mathrm{~mL} / \mathrm{min}$ per $1.73 \mathrm{~m}^{2}$ or urine albumin: creatinine ratio $3 \mathrm{mg} / \mathrm{mmol}$ or greater. ${ }^{21} \mathrm{We}$ identified patients with hypertension through prescriptions for antihypertensive medications. Patients with diabetes were identified by an $\mathrm{HbA}_{1 \mathrm{c}}$ concentration of $6.5 \%$ or less, or the use of a diabetic medication. We identified patients with dyslipidemia by elevated LDL-C level $(\geq 2.0 \mathrm{mmol} / \mathrm{L})$ or the use of a lipidlowering medication. ${ }^{12}$

\section{Statistical analysis}

We calculated the prevalence of chronic kidney disease in patients with at least 1 albumin:creatinine ratio or estimated glomerular filtration rate investigation during the study period, age adjusted to the 2016 Canadian census population. We calculated the comorbid prevalence of diabetes, hypertension and dyslipidemia using the population of patients with chronic kidney disease as the denominator. We calculated the presence of multiple comorbidities as the proportion of patients with chronic kidney disease affected. Data are presented as mean and standard deviation for continuous variables and proportions for discreet variables.

\section{Ethics approval}

The research was supervised and the data were maintained by the Sioux Lookout First Nations Health Authority, which represents communities in health programming and surveillance in accordance with the principles of ownership, control, access and possession (OCAP). ${ }^{22}$ Ethics approval was granted by the Sioux Lookout Meno Ya Win Research Review and Ethics Committee and the Lakehead University Research Ethics Board.

\section{Results}

The total population of northwest Ontario in 2017 was 24789 , including 16170 adults residing in the 26 communities. ${ }^{17}$ Renal testing (estimated glomerular filtration rate or albumin:creatinine ratio) had been performed in 5224 unique patients $(32.3 \%)$ from May 2014 to May 2017. Of the 16170 adults, 4578 (28.3\%) had at least 1 estimated glomerular filtration rate measurement, and $2462(15.2 \%)$ at least 1 albumin:creatinine ratio measurement, with overlap between these 2 groups. Abnormal results occurred in $806(17.6 \%)$ of the estimated glomerular filtration rate tests and $1433(58.2 \%)$ of the albumin:creatinine ratio tests.

We identified 1859 patients with chronic kidney disease. The resulting age-adjusted chronic kidney disease prevalence was $14.7 \%$. The mean age of these patients was 55 years, 14 years older than the average adult in the electronic medical record (Table 1). Of the 5224 adults who had renal testing, 2945 (56.4\%) were women, and they had a burden of chronic kidney disease similar to that of men. The age-adjusted prevalence of reduced renal function (stage 3-5 chronic kidney disease, estimated glomerular filtration rate $<60 \mathrm{~mL} / \mathrm{min}$ ) was $7.0 \%$, and that of albuminuria with normal renal function (stage 1-2 chronic kidney disease) was $7.7 \%$ (Table 2).

A total of 1487 patients with chronic kidney disease $(80.0 \%)$ had at least 1 cardiovascular comorbidity (diabetes, hypertension or dyslipidemia), 1078 (58.0\%) had 2 cardiovascular comorbidities, and 716 (38.5\%) had all 3 cardiovascular comorbidities (Table 3). Diabetes was most often coprevalent (1332 [71.6\%]), followed by dyslipidemia (1313 [70.6\%]) and hypertension (1098 [59.1\%]).

Almost one-third (539 [29.0\%]) of patients with chronic kidney disease were not screened for lipid levels during the study period, $980(52.7 \%)$ were receiving a statin, and 688 (37.0\%) met the recommended LDL-C goal.

\begin{tabular}{|c|c|c|c|}
\hline Characteristic & $\begin{array}{c}\text { Total } \\
\text { population } \\
\text { aged } \geq 18 \mathrm{yr} \\
n=16170\end{array}$ & $\begin{array}{c}\text { Population } \\
\text { tested } \\
n=5224\end{array}$ & $\begin{array}{c}\text { Patients with } \\
\text { chronic } \\
\text { kidney } \\
\text { disease } \\
n=1859\end{array}$ \\
\hline Female sex, no. (\%) & $8048(49.8)$ & $2945(56.4)$ & $1021(54.9)$ \\
\hline Age, yr, mean \pm SD & $41 \pm 16.9$ & $50 \pm 16.0$ & $55 \pm 16.0$ \\
\hline
\end{tabular}

Note: SD = standard deviation 


\section{Table 2: Prevalence of chronic kidney disease}

\begin{tabular}{|lcc|}
\hline Severity of disease & Crude prevalence (\%) & $\begin{array}{c}\text { Age-adjusted } \\
\text { prevalence, }\end{array}$ \\
\hline $\begin{array}{l}\text { Stage 1-2 } \\
\text { + albuminuria }\end{array}$ & $1053 / 16170(6.5)$ & 7.7 \\
\hline $\begin{array}{l}\text { Stage 3-5 } \\
\pm \text { albuminuria }\end{array}$ & $806 / 16170(5.0)$ & 7.0 \\
\hline Total & $1859 / 16170(11.5)$ & 14.7 \\
\hline *Adjusted to 2016 Canadian census population. \\
\hline
\end{tabular}

Table 3: Laboratory values, drug use and comorbidities of patients with chronic kidney disease

\begin{tabular}{|c|c|}
\hline Variable & $\begin{array}{l}\text { No. }(\%) \text { of } \\
\text { patients* } \\
n=1859\end{array}$ \\
\hline \multicolumn{2}{|l|}{ Laboratory values, mean \pm SD } \\
\hline Glycated hemoglobin concentration & $8.3 \pm 2.3$ \\
\hline $\begin{array}{l}\text { Low-density lipoprotein cholesterol level, } \\
\mathrm{mmol} / \mathrm{L}\end{array}$ & $2.1 \pm 0.6$ \\
\hline \multicolumn{2}{|l|}{ Drug use } \\
\hline Statin & $980(52.7)$ \\
\hline $\begin{array}{l}\text { Angiotensin-converting-enzyme inhibitor } \\
\text { or angiotensin receptor blocker }\end{array}$ & 619 (33.3) \\
\hline \multicolumn{2}{|l|}{ Comorbidities } \\
\hline Diabetes mellitus & $1332(71.6)$ \\
\hline Dyslipidemia & $1313(70.6)$ \\
\hline Hypertension & $1098(59.1)$ \\
\hline \multicolumn{2}{|l|}{2 comorbidities $†$} \\
\hline Diabetes + dyslipidemia & $1069(57.5)$ \\
\hline Diabetes + hypertension & 877 (47.2) \\
\hline Hypertension + dyslipidemia & $852(45.8)$ \\
\hline \multicolumn{2}{|l|}{3 comorbidities $\dagger$} \\
\hline Hypertension + diabetes + dyslipidemia & $716(38.5)$ \\
\hline $\begin{array}{l}\text { Note: } \mathrm{SD}=\text { standard deviation. } \\
\text { "Except where noted otherwise. } \\
\text { †Some patients are represented in more than } 1 \text { group. }\end{array}$ & \\
\hline
\end{tabular}

\section{Interpretation}

Renal screening occurred in $32 \%$ of the adult population of 26 First Nations communities of northwest Ontario during the 3 -year study period; $15 \%$ had albumin:creatinine ratio testing, and $28 \%$ had estimated glomerular filtration rate testing. There was a high coprevalence of diabetes (72\%), dyslipidemia $(71 \%)$ and hypertension (59\%). A substantial proportion of patients with chronic kidney disease, $38 \%$, had all 3 cardiovascular comorbidities. The age-adjusted prevalence of chronic kidney disease was $14.7 \%$; half of these patients had stage 3-5 disease.
The prevalence of chronic kidney disease in our population is slightly higher than the 2013 estimated Canadian rate of $12.5 \%{ }^{23}$ but lower than that in recent population screening reports among Manitoba First Nations and southern Ontario First Nations communities of $25 \%$ and $30 \%$, respectively. ${ }^{47,23}$ Our prevalence of stage 3-5 chronic kidney disease is higher, at $7 \%$ versus $3 \%-5 \%,{ }^{5,6,23}$ These variations are likely due to methodological differences, primarily the use of a screened population versus a practice-based cohort. Studies using data arising from patient care cohorts showed a higher prevalence of stage 3-5 disease (7\%-8\%). ${ }^{14,24}$ Our methodology approximated that of a 2019 study of the prevalence of advanced chronic kidney disease in the general Canadian population, with electronic medical record data extraction from primary care practices. ${ }^{24}$ Those authors used only estimated glomerular filtration rate results (no albumin:creatinine ratio), with findings equivalent to ours (prevalence of stage 3-5 disease of $7 \%$ ). Similarly, in a study using data from health administrative databases from 1980-2005 (before the advent of the electronic medical record) in Saskatchewan, the prevalence of advanced disease was $8 \% .{ }^{14}$

The high coprevalence of chronic kidney disease and diabetes in our population, $72 \%$, is not surprising given the high burden of diabetes in First Nations populations. This rate is higher than that reported in a recent Manitoba screening study, $60 \% .5,25,26$ The coprevalence of dyslipidemia, $71 \%$, is higher than estimates of $30 \%$ in the overall adult First Nations and Canadian populations. ${ }^{27,28}$ The average LDL-C level in our patients with chronic kidney disease was $2.1 \mathrm{mmol} / \mathrm{L}$, lower than the value of $2.4 \mathrm{mmol} / \mathrm{L}$ observed in the 2012 Canadian First Nations Diabetes Clinical Management and Epidemiologic (CIRCLE) study among 885 First Nations people with diabetes. ${ }^{4}$ The coprevalence of hypertension in our population, $59 \%$, is higher than the rates of $27 \%$ identified in the 2016 Manitoba study ${ }^{5}$ and $29.5 \%$ in a 2011 study of 555 First Nations community members in southern Ontario. ${ }^{6}$ Only $56 \%$ of our patients with chronic kidney disease who had hypertension were receiving treatment with angiotensin-converting-enzyme inhibitors or angiotensin receptor blockers. The prevalence of these comorbidities with chronic kidney disease confers additional cardiovascular risk. ${ }^{13,14}$ The CIRCLE study investigators noted that progression from albuminuria to advanced chronic kidney disease was associated with duration of diabetes and coprevalence of hypertension and dyslipidemia in First Nations patients. ${ }^{4}$

Chronic kidney disease independently increases the risk of adverse cardiovascular events. ${ }^{29,30}$ The high coprevalence of other cardiovascular comorbidities in this First Nations population signals the need for broad assessment and risk management. ${ }^{14,30}$ These findings will play an important role in planning dialysis services and in secondary prevention of progression of chronic kidney disease and cardiovascular disease.

\section{Limitations}

Our study has limitations owing to the real-world nature of the data, with relevant testing of only $32 \%$ of the adult population. Our noted prevalence of chronic kidney disease is 
likely an underestimate when compared to screening studies that include universal albumin:creatinine ratio and estimated glomerular filtration rate testing. ${ }^{5,6}$ Urine albumin:creatinine ratio testing gives the highest yield of chronic kidney disease detection but is not universally used in general practice; it was ordered in only $15 \%$ of our population. Urine screening at point-of care-testing (dipstick) is common but is not systematically recorded in this electronic medical record (available in clinical notes only). Our study is therefore limited in identifying early-stage chronic kidney disease, but the high prevalence of advanced chronic kidney disease in this population is notable, particularly as only $28 \%$ of patients received estimated glomerular filtration rate testing. Retrospective data collection based on electronic medical record is limited to available and extractable data. Information on blood pressure measurements, body mass index, and rates of smoking and cardiovascular disease were not available, nor were diagnosis or physician billing data.

\section{Conclusion}

We document a substantial prevalence of stage 3-5 chronic kidney disease and associated cardiovascular comorbidities in this First Nations population. Coprevalence of these major chronic diseases in this population increases cardiovascular risk, and robust clinical assessment is warranted. These findings will help health care providers identify people at high risk for cardiovascular and progressive kidney disease.

\section{References}

1. Neovius M, Jacobson SH, Eriksson JK, et al. Mortality in chronic kidney disease and renal replacement therapy: a population-based cohort study. BMF Open 2014;4:e004251.

2. Zelmer JL. The economic burden of end-stage renal disease in Canada. Kidney Int 2007;72:1122-9.

3. Finnegan-John J, Thomas VJ. The psychosocial experience of patients with end-stage renal disease and its impact on quality of life: findings from a needs assessment to shape a service. ISRN Nephrol 2012;2013:308986.

4. Dyck RF, Hayward MN, Harris SB; CIRCLE Study Group. Prevalence, determinants and co-morbidities of chronic kidney disease among First Nations adults with diabetes: results from the CIRCLE study. BMC Nephrol 2012;13:57.

5. Komenda P, Lavallee B, Ferguson TW, et al. The prevalence of CKD in rural Canadian Indigenous peoples: results from the First Nations Community Based Screening to Improve Kidney Health and Prevent Dialysis (FINISHED) screen, triage, and treat program. Am F Kidney Dis 2016;68:582-90.

6. Ashton CW, Duffie D. Chronic kidney disease in Canada's First Nations: results of an effective cross-cultural collaboration. Healthc Q 2011;14:42-7.

7. Anand SS, Yusuf S, Jacobs R, et al. Risk factors, atherosclerosis, and cardiovascular disease among Aboriginal people in Canada: the Study of Health Assessment and Risk Evaluation in Aboriginal Peoples (SHARE-AP). Lancet 2001; 358:1147-53

8. Johnson JA, Vermeulen SU, Toth EL, et al. Increasing incidence and prevalence of diabetes among the Status Aboriginal population in urban and rural Alberta, 1995-2006. Can 7 Public Health 2009;100:231-6.

9. Bruce SG, Riediger ND, Zacharias JM, et al. Obesity and obesity-related comorbidities in a Canadian First Nation population. Chronic Dis Can 2010;31:27-32.

10. Riediger ND, Bruce SG, Young TK. Cardiovascular risk according to plasma apolipoprotein and lipid profiles in a Canadian First Nation. Cbronic Dis Can 2010;31:33-8.

11. Anand SS, Yi Q, Gerstein H, et al.; Study of Health Assessment and Risk in Ethnic Groups; Study of Health Assessment and Risk Evaluation in Aboriginal Peoples Investigators. Relationship of metabolic syndrome and fibrinolytic dysfunction to cardiovascular disease. Circulation 2003;108:420-5.

12. Anderson TJ, Grégoire J, Pearson GJ, et al. 2016 Canadian Cardiovascular Society guidelines for the management of dyslipidemia for the prevention of cardiovascular disease in the adult. Can 7 Cardiol 2016;32:1263-82.

13. Gao S, Manns BJ, Culleton BF, et al.; Alberta Kidney Disease Network. Access to health care among status Aboriginal people with chronic kidney disease. CMA7 2008;179:1007-12.
14. Dyck RF, Jiang Y, Osgood ND. The long-term risks of end stage renal disease and mortality among First Nations and non-First Nations people with youthonset diabetes. Can $\mathcal{f}$ Diabetes 2014;38:237-43.

15. Greiver M, Williamson T, Bennett TL, et al.; Canadian Primary Care Sentinel Surveillance Network. Developing a method to estimate practice denominators for a national Canadian electronic medical record database. Fam Pract 2013;30: 347-54.

16. Sibley LM, Moineddin R, Ahga MM, et al. Risk adjustment using administrative data-based and survey-derived methods for explaining physician utilization. Med Care 2010;48:175-82.

17. Annual report 2017/2018. Sioux Lookout (ON): Sioux Lookout First Nations Health Authority. Available: www.slfnha.com/application/files/5715/3754/2904/ SLFNHA_Annual_Report_2017-18_FINAL_WEB.pdf (accessed 2019 July 5).

18. Williamson T, et al. CPCSSN disease definitions. Kingston (ON): Canadian Primary Care Sentinel Surveillance Network; 2014 June 15. Available: http:// cpcssn.ca/research-resources/case-definitions (accessed 2019 July 5).

19. Williamson T, Green ME, Birthwhistle R, et al. Validating the 8 CPSSN case definitions for chronic disease in a primary care database of electronic health records. Ann Fam Med 2014;12:367-72.

20. Guidelines for ATC classification and DDD assignment 2019. Oslo: WHO Collaborating Centre for Drug Statistics Methodology; 2018. Available: www.whocc. no/filearchive/publications/2019_guidelines_web.pdf (accessed 2019 Feb. 27).

21. KDIGO 2012 clinical practice guidelines for the evaluation and management of chronic kidney disease. Kidney Int Suppl 2013;3:1-150.

22. The First Nations principles of OCAP®. Ottawa: First Nations Information Governance Centre. Available: https://fnigc.ca/ocap (accessed 2019 June 26).

23. Arora P, Vasa P, Brenner D, et al. Prevalence estimates of chronic kidney disease in Canada: results of a nationally representative survey. CMAJ 2013;185:E417-23.

24. Bello AK, Ronksley PE, Tangri N, et al. Prevalence and demographics of CKD in Canadian primary care practices: a cross-sectional study. Kidney Int Rep 2019;4:561-70.

25. Naqshbandi M, Harris SB, Esler JG, et al. Global complication rates of type 2 diabetes in Indigenous peoples: a comprehensive review. Diabetes Res Clin Pract 2008;82:1-17.

26. Hayward MN, Harris SB, Esler J, et al. Results of a pilot national diabetes surveillance system for First Nations [abstract]. Can 7 Diabetes 2012;36(Suppl):S21.

27. Riediger ND, Lukianchuk V, Bruce SG. Incident diabetes, hypertension and dyslipidemia in a Manitoba First Nation. Int 7 Circumpolar Health 2015;74:27712.

28. Asghari S, Aref-Eshghi E, Godwin M, et al. Single and mixed dyslipidaemia in Canadian primary care settings: findings from the Canadian Primary Care Sentinel Surveillance Network database. BMF Open 2015;5:e007954.

29. Said S, Hernandez GT. The link between chronic kidney disease and cardiovascular disease. 7 Nephropathol 2014;3:99-104.

30. Hemmelgarn BR, Manns BJ, Lloyd A, et al.; Alberta Kidney Disease Network. Relation between kidney function, proteinuria, and adverse outcomes. $7 A M A$ 2010;303:423-9.

Affiliations: Anishinaabe Bimaadiziwin Research Program (Kelly, Schreiber, Willms), Sioux Lookout Meno Ya Win Health Centre; Approaches to Community Wellbeing (Matsumoto) and Health Services (Gordon, Hopko), Sioux Lookout First Nations Health Authority, Sioux Lookout, Ont.; Division of Infectious Diseases (Schreiber), The Ottawa Hospital, University of Ottawa; The Ottawa Hospital Research Institute (Schreiber); School of Epidemiology and Public Health (Schreiber), University of Ottawa, Ottawa, Ont.; University of Saskatchewan (Olivier), Saskatoon, Sask.; Division of Clinical Sciences (Madden), Northern Ontario School of Medicine, Sioux Lookout, Ont.; Department of Medicine (Tobe), Sunnybrook Health Sciences Centre, Toronto, Ont.

Contributors: Sheldon Tobe initiated the study. Len Kelly designed the study. Janet Gordon and Sheldon Tobe oversaw the study, and Janet Gordon oversaw the study process, including community permission and data extraction. Cai-lei Matsumoto supervised the data collection and analyzed the data. Christopher Olivier and Josh Hopko collected the data. Hannah Willms and Christopher Olivier performed the literature searches. Len Kelly, Sheldon Tobe and Cai-lei Matsumoto interpreted the data. Len Kelly, Yoko Schreiber, Sharen Madden and Sheldon Tobe drafted the manuscript, and Yoko Schreiber and Sharen Madden critically revised it for important intellectual content. All of the authors approved the final version to be published and agreed to act as guarantors of the work.

Funding: This work was supported by the Northern Ontario Academic Medicine Association.

Supplemental information: For reviewer comments and the original submission of this manuscript, please see www.cmajopen.ca/content/7/3/ E568/suppl/DC1. 